\title{
Improved Formulations of the Superposition Method
}

\author{
Lian-Ping Wang and Orlando Ayala \\ Department of Mechanical Engineering, University of Delaware, Newark, Delaware \\ WOJCIECH W. GRABOWSKI \\ Mesoscale and Microscale Meteorology Division, National Center for Atmospheric Research,* Boulder, Colorado
}

(Manuscript received 20 January 2004, in final form 15 July 2004)

\begin{abstract}
Two formulations of an improved superposition method are proposed for studying droplet-droplet hydrodynamic interactions. The formulations make explicit use of the boundary conditions on the surface of the two interacting droplets. The improved formulations are described through a consistent and rigorous consideration of the relationship between the drag force and representation of disturbance flows. It is demonstrated that the improved formulations are much more accurate than the original implementation of the superposition method. Specifically, for the case of Stokes disturbance flows, the relative errors on the drag force can be reduced by one order of magnitude using the improved formulations, when compared with the original formulation, for situations when the lubrication effect is not dominant. Using the improved superposition method, collision efficiencies of small cloud droplets falling in calm air are also computed and compared with previously published results.
\end{abstract}

\section{Introduction}

The topic of collision coalescence of cloud droplets is of great importance to the understanding and quantitative prediction of warm rain formation (Pruppacher and Klett 1997). For droplets of radii less than $60 \mu \mathrm{m}$, hydrodynamic interactions between two colliding droplets can significantly affect the trajectories of the droplets and therefore modify the overall collision efficiency (e.g., Klett and Davis 1973). This may be explained qualitatively as follows. A small droplet of finite size moving in a fluid medium introduces a disturbance flow field in its neighborhood and as such modifies the flow field locally. For droplets of radii less than $60 \mu \mathrm{m}$, the hydrodynamic interaction time (say, the ratio of collision radius over differential settling velocity) may be larger than the inertial response times of the droplets, so at least one of the droplets has adequate time to respond to the disturbance flow induced by the other droplet. The collision radius here is the geometric col-

\footnotetext{
* The National Center for Atmospheric Research is sponsored by the National Science Foundation.
}

Corresponding author address: Lian-Ping Wang, Department of Mechanical Engineering, 126 Spencer Laboratory, University of Delaware, Newark, DE 19716-3140.

E-mail: lwang@me.udel.edu lision radius defined as the sum of the radii of the two colliding droplets.

A representation of these disturbance flows is then needed for quantitative prediction of collision efficiency, which is a required input for the modeling of size distribution of cloud droplets through collision coalescence. One well-known approximate approach is the superposition method (see, e.g., Pruppacher and Klett 1997). The method was initially designed to study the hydrodynamic interaction of two spherical droplets using the solution of disturbance flow induced by a single sphere. Basically, it is assumed that each droplet moves in a flow field generated by the other droplet falling in isolation. The method, because of its simplicity, has been widely used for treating hydrodynamic interactions of droplets under both the Stokes disturbance flows (Langmuir 1948; Pinsky et al. 1999) and disturbance flows at finite Reynolds numbers (Beard and Grover 1974; Neiburger 1967; Shafrir and Neiburger 1963; Lin and Lee 1975).

It is known that the superposition method becomes inaccurate when the separation distance between two droplets is comparable to the collision radius (Klett 1976; Pruppacher and Klett 1997). As we point out in this paper, this inaccuracy is not a necessary consequence of the superposition method. We shall demonstrate that most of this inaccuracy can be removed by simply revising the way that the perturbation velocities at the locations of droplets are computed. Our proposal 
is to impose proper boundary conditions at the surface of the droplets, which provides a natural optimization procedure for the superposition method. The idea is similar to that of Klett and Davis (1973) who superimposed two Oseen flows while requiring the composite flow to satisfy the necessary boundary conditions. The theoretical framework discussed in this paper, however, is more consistent as far as Stokes disturbance flows are considered and is described more thoroughly than that of Klett and Davis (1973).

For simplicity, we treat the droplets as solid, nonrotating spherical particles with nonslip surface boundary conditions. Only Stokes disturbance flows are considered. It should be pointed out that the no-slip boundary condition is no longer valid when the minimum separation between two droplets approaches the mean free path of air molecules, as the physical assumption that the fluid acts as a continuum breaks down (Hocking 1973; Jonas 1972). Or alternatively, the lubrication force between droplets at close separations are typically overestimated by the no-slip boundary condition, leading to underestimation of collision efficiencies and strong dependence of collision efficiencies on the minimum separation gap used to define collisions (Hocking and Jonas 1970; Jonas 1972; Hocking 1973). As will be seen later in this paper, all formulations of the superposition method are unable to handle the lubrication effect. This, however, may be viewed to our advantage in the sense that our results of collision efficiencies are not sensitive to the minimum separation gap used for collision detection.

In section 2, we present a formulation of the improved superposition method. In section 3, the results of the improved superposition method are then compared with those based on the original implementation of the superposition method as well as known analytical results of Stimson and Jeffrey (1926) and Davis (1969). These include drag forces on the droplets, flow visualization, and collision efficiencies of small cloud droplets falling in calm air. Finally, conclusions are drawn in section 4 .

\section{Formulation of an improved superposition method}

We first reexamine the original formulation of the superposition method by questioning what is exactly the representation of the disturbance flows that led to the modified drag force representation used in the method. For this purpose, we need to review some basic properties of a Stokes flow.

\section{a. Basic properties of Stokes disturbance flow induced by a moving sphere}

We first consider the problem of a single droplet moving at a constant velocity $\mathbf{V}$ in still air. The direction of $\mathbf{V}$ may not be the same as gravity (Fig. 1). The drop-

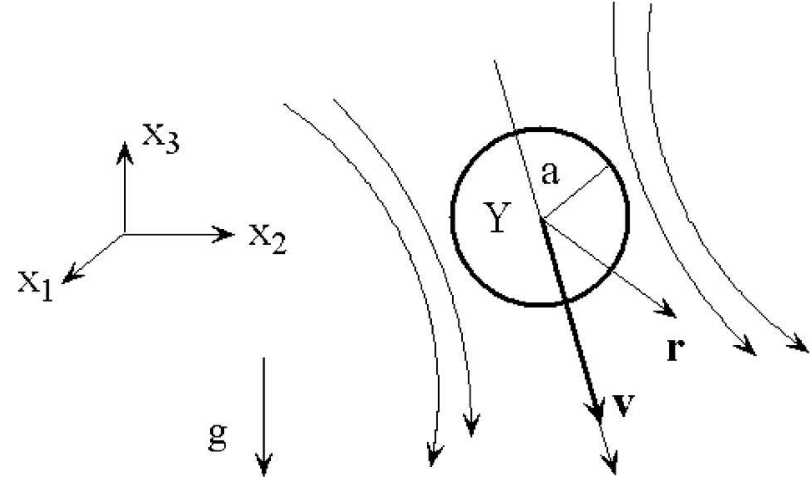

FIG. 1. Notation for a single droplet.

let will introduce a disturbance airflow field. Assuming that the Reynolds number based on $|\mathbf{V}|$ and droplet diameter is very small, the unsteady Stokes disturbance flow induced by the droplet may be written in a vector form (Kim and Karrila 1991):

$$
\begin{aligned}
\mathbf{u}(\mathbf{x}, t)= & {\left[\frac{3}{4} \frac{a}{r}-\frac{3}{4}\left(\frac{a}{r}\right)^{3}\right] \frac{\mathbf{r}}{r^{2}}(\mathbf{V} \cdot \mathbf{r}) } \\
& +\left[\frac{3}{4} \frac{a}{r}+\frac{1}{4}\left(\frac{a}{r}\right)^{3}\right] \mathbf{V} \equiv \mathbf{u}_{s}(\mathbf{r} ; a, \mathbf{V}),
\end{aligned}
$$

where $a$ is the droplet radius, and $\mathbf{r}$ is the position vector relative to the center of the droplet $\mathbf{Y}(t)=\mathbf{Y}(0)+\mathbf{V} t$,

$$
\mathbf{r}(\mathbf{x}, t)=\mathbf{x}-\mathbf{Y}(t)=\mathbf{x}-\mathbf{Y}(0)-\mathbf{V} t .
$$

The above vector form will facilitate the description of the two-droplet problem to be discussed later. Equations (1) and (2) imply that

$$
[\partial \mathbf{u} / \partial t+\mathbf{V} \cdot \nabla \mathbf{u}]=0
$$

namely, the Stokes flow pattern is advected with the droplet velocity $\mathbf{V}$.

The induced pressure field can be expressed as (Kim and Karrila 1991)

$$
p(\mathbf{x}, t)=\frac{3 a \mu}{2 r^{3}} \mathbf{V} \cdot \mathbf{r} \equiv p_{s}(\mathbf{r} ; a, \mathbf{V}),
$$

where $\mu$ is the fluid dynamic viscosity.

It is straightforward to show that the above disturbance flow field and pressure field satisfy the following unsteady Stokes equation with a linearized advection term:

$$
\rho\left[\frac{\partial \mathbf{u}}{\partial t}+\mathbf{V} \cdot \nabla \mathbf{u}\right]=-\nabla\left(p+p_{0}\right)+\mu \nabla^{2} \mathbf{u}-\rho g \mathbf{e}_{3},
$$

where $p_{0}(\mathbf{x}, t)=-\rho g x_{3}$ is the hydrostatic pressure field, $\rho$ is the air density, $x_{3}$ is the vertical spatial coordinate pointing upward, $g$ is the gravitational acceleration, and $\mathbf{e}_{3}$ is the unit vector in the $x_{3}$ direction. The linearized advection term is a good approximation to the true advection term very close to the surface of the droplet.

In fact, the two terms on the left-hand side of the 
above equation sum up exactly to zero due to (3), with each of them individually scaled as $\rho V^{2} / a$ close to the moving droplet. The terms $\nabla p$ and $\mu \nabla^{2} \mathbf{u}$ are each scaled as $\mu V / a^{2}$, and, therefore, are much larger than the terms on the left-hand side if the droplet Reynolds number $\operatorname{Re}=\rho V(2 a) / \mu$ is much less than one. Therefore, to the leading order and when $(\operatorname{Re} r / a)<1$ the following quasi-steady Stokes equation can replace (5):

$$
-\nabla\left(p+p_{0}\right)+\mu \nabla^{2} \mathbf{u}-\rho g \mathbf{e}_{3}=0 .
$$

The above quasi-steady Stokes flow representation may also be viewed as a leading-order solution to the problem of a droplet moving at time-dependent velocity $\mathbf{V}(t)$, provided that, in addition to the condition of a very small droplet Reynolds number, the viscous diffusion time scale, $\rho a^{2} / \mu$, is much less than the characteristic time scale associated with $\mathbf{V}(t)$, namely, the droplet response time $\tau_{p}=2 \rho_{p} a^{2} /(9 \mu)$, where $\rho_{p}$ is the droplet density. This additional condition amounts to $\rho_{p} / \rho \gg$ 4.5 , which is easily satisfied in the context of cloud microphysics. Therefore, we could extend the use of quasi-steady Stokes disturbance flow for any timedependent motion of small cloud droplets. A very similar argument was made in previous publications to neglect the fluid acceleration (e.g., Davis 1969).

Of importance is the fact that the disturbance flow given by Eq. (1) satisfies the boundary condition on the surface of the droplet:

$$
\left.\mathbf{u}(\mathbf{x}, t)\right|_{|\mathbf{r}|=a}=\mathbf{V} .
$$

The total force acting on the surface of the droplet can be calculated as

$$
F_{i}=\oint_{|\mathbf{r}|=a}\left[-\left(p+p_{0}\right) \delta_{i j}+\mu\left(\frac{\partial u_{i}}{\partial x_{j}}+\frac{\partial u_{j}}{\partial x_{i}}\right)\right] n_{j} d S,
$$

leading to the well-known result,

$$
\mathbf{F}=\rho g \frac{4 \pi a^{3}}{3} \mathbf{e}_{3}-6 \pi \mu a \mathbf{V}
$$

where the first term is the buoyancy force (typically neglected in cloud physics because of the density ratio between water and air), and the second term is the Stokes drag.

If an imaginary spherical surface $S_{I}$ of center $\mathbf{x}_{I}$ and radius $b$ is introduced outside the droplet, then the total force acting on this imaginary surface due to the same Stokes disturbance flow can be shown to be

$$
\begin{gathered}
\mathbf{F}_{I}=\oint_{\left|\mathbf{x}-\mathbf{x}_{I}\right|=b}\left[-\left(p+p_{0}\right) \delta_{i j}+\mu\left(\frac{\partial u_{i}}{\partial x_{j}}+\frac{\partial u_{j}}{\partial x_{i}}\right)\right] \\
\times n_{j} d S=\rho g \frac{4 \pi b^{3}}{3} \mathbf{e}_{3} .
\end{gathered}
$$

Namely, the disturbance flow does not result in any viscous force, since the imaginary surface does not con- tain any singularity of the disturbance flow field (Kim and Karrila 1991).

\section{b. Superposition method for the two-droplet problem}

Now consider two droplets moving in still air. The droplet radii are $a_{1}$ and $a_{2}$, center positions are $\mathbf{Y}_{1}$ and $\mathbf{Y}_{2}$, and velocities are $\mathbf{V}_{1}$ and $\mathbf{V}_{2}$ (Fig. 2). The induced flow due to the second droplet moving in isolation is $\mathbf{u}_{s}\left(\mathbf{r}_{2} ; a_{2}, \mathbf{V}_{2}\right)$. The original implementation of the superposition method assumes that the net drag force acting on the surface of the first droplet is

$$
\mathbf{D}_{1}=-6 \pi \mu a_{1}\left[\mathbf{V}_{1}-\mathbf{u}_{s}\left(\mathbf{Y}_{1}-\mathbf{Y}_{2} ; a_{2}, \mathbf{V}_{2}\right)\right] \text {. }
$$

Similarly, the net drag force on the second droplet is

$$
\mathbf{D}_{2}=-6 \pi \mu a_{2}\left[\mathbf{V}_{2}-\mathbf{u}_{s}\left(\mathbf{Y}_{2}-\mathbf{Y}_{1} ; a_{1}, \mathbf{V}_{1}\right)\right]
$$

In other words, the velocity of each droplet relative to air is reduced by the Stokes flow solution due to the presence of the other droplet.

Based on the properties of the Stokes flow discussed above, the above drag force representations imply a composite disturbance flow field as follows:

$$
\begin{aligned}
\mathbf{u}(\mathbf{x}, t)= & \mathbf{u}_{s}\left[\mathbf{r}_{1} ; a_{1}, \mathbf{V}_{1}-\mathbf{u}_{s}\left(\mathbf{Y}_{1}-\mathbf{Y}_{2} ; a_{2}, \mathbf{V}_{2}\right)\right] \\
& +\mathbf{u}_{s}\left[\mathbf{r}_{2} ; a_{2}, \mathbf{V}_{2}-\mathbf{u}_{s}\left(\mathbf{Y}_{2}-\mathbf{Y}_{1} ; a_{1}, \mathbf{V}_{1}\right)\right]
\end{aligned}
$$

where $\mathbf{r}_{1}=\mathbf{x}-\mathbf{Y}_{1}(t)$ and $\mathbf{r}_{2}=\mathbf{x}-\mathbf{Y}_{2}(t)$. Although this composite disturbance flow satisfies the Stokes Eq. (6), it does not satisfy the two boundary conditions (BCs) on the surface of the droplets: $\left.\mathbf{u}(\mathbf{x}, t)\right|_{\left|\mathbf{r}_{1}\right|=a_{1}}=\mathbf{V}_{1}$ and $\left.\mathbf{u}(\mathbf{x}, t)\right|_{\left|\mathbf{r}_{2}\right|=a_{2}}=\mathbf{V}_{2}$. (An example of the composite flow is given later in Fig. 4a to show that the airflow cuts through the boundaries of the droplets with the above

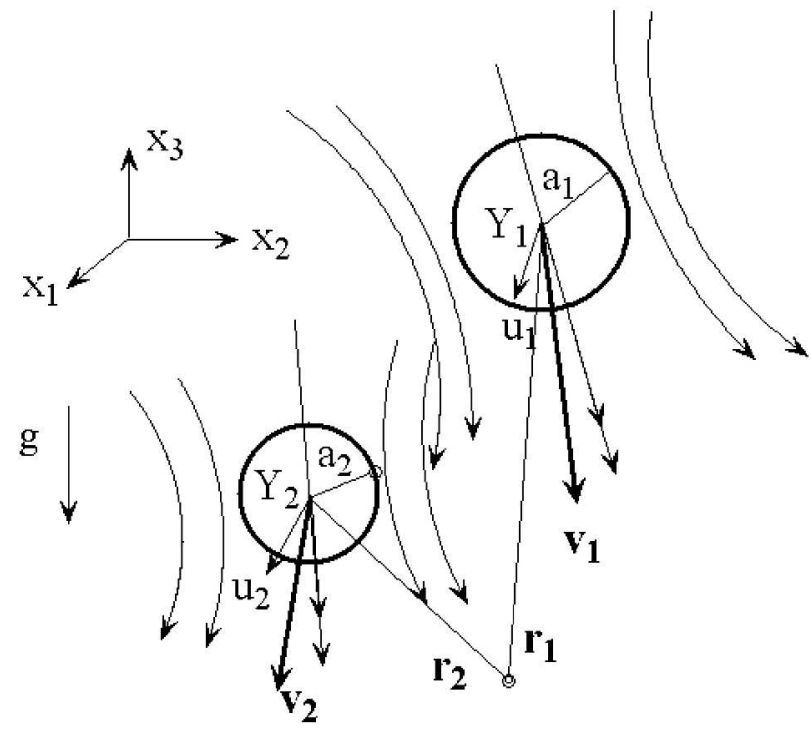

FIG. 2. Notation for the two-droplet problem. 
original formulation of the superposition method, and further that the average air velocity on the surface of each droplet is significantly different from the velocity of the droplet.)

\section{c. The improved superposition method}

The boundary conditions on the surface of droplets can be better satisfied by assuming a composite disturbance flow field:

$$
\mathbf{u}(\mathbf{x}, t)=\mathbf{u}_{s}\left(\mathbf{r}_{1} ; a_{1}, \mathbf{V}_{1}-\mathbf{u}_{1}\right)+\mathbf{u}_{s}\left(\mathbf{r}_{2} ; a_{2}, \mathbf{V}_{2}-\mathbf{u}_{2}\right) .
$$

Here $\mathbf{u}_{1}$ and $\mathbf{u}_{2}$ are the levels of disturbance flow due to the presence of the other droplets that need to be determined. The alignment of streamlines of each disturbance flow is illustrated in Fig. 2. We will impose the condition that the average fluid velocity on the surface of each droplet is equal to the velocity of that droplet (no slip), namely,

$$
\begin{aligned}
& \mathbf{V}_{1}=\mathbf{V}_{1}-\mathbf{u}_{1}+\frac{1}{4 \pi a_{1}^{2}} \oint_{\left|\mathbf{r}_{1}\right|=a_{1}} \mathbf{u}_{s}\left(\mathbf{r}_{2} ; a_{2}, \mathbf{V}_{2}-\mathbf{u}_{2}\right) d s, \\
& \mathbf{V}_{2}=\mathbf{V}_{2}-\mathbf{u}_{2}+\frac{1}{4 \pi a_{2}^{2}} \oint_{\left|\mathbf{r}_{2}\right|=a_{2}} \mathbf{u}_{s}\left(\mathbf{r}_{1} ; a_{1}, \mathbf{V}_{1}-\mathbf{u}_{1}\right) d s .
\end{aligned}
$$

Or equivalently, we have the following integral equations to solve $\mathbf{u}_{1}$ and $\mathbf{u}_{2}$ :

$$
\begin{aligned}
& \mathbf{u}_{1}=\frac{1}{4 \pi a_{1}^{2}} \oint_{\left|\mathbf{r}_{1}\right|=a_{1}} \mathbf{u}_{s}\left(\mathbf{r}_{2} ; a_{2}, \mathbf{V}_{2}-\mathbf{u}_{2}\right) d s \\
& \mathbf{u}_{2}=\frac{1}{4 \pi a_{2}^{2}} \oint_{\left|\mathbf{r}_{2}\right|=a_{2}} \mathbf{u}_{s}\left(\mathbf{r}_{1} ; a_{1}, \mathbf{V}_{1}-\mathbf{u}_{1}\right) d s
\end{aligned}
$$

The above integral equation may be solved exactly if the integrations can be performed. Alternatively, if we evaluate the integrands using the midpoint values (i.e., at the center of droplets), we have the following approximations:

$$
\begin{aligned}
& \mathbf{u}_{1}=\mathbf{u}_{S}\left(\mathbf{Y}_{1}-\mathbf{Y}_{2} ; a_{2}, \mathbf{V}_{2}-\mathbf{u}_{2}\right), \\
& \mathbf{u}_{2}=\mathbf{u}_{S}\left(\mathbf{Y}_{2}-\mathbf{Y}_{1} ; a_{1}, \mathbf{V}_{1}-\mathbf{u}_{1}\right) .
\end{aligned}
$$

For the two-droplet problem, $\mathbf{u}_{1}$ and $\mathbf{u}_{2}$ can be solved directly since the Stokes flow is linear. The physical interpretation of $\mathbf{u}_{1}$ in the integral formulation, (17), is the average perturbation velocity on the surface of the first droplet, induced by the second droplet. In the second, center-point formulation, (19), $\mathbf{u}_{1}$ is the perturbation velocity at the center of droplet 1 , induced by droplet 2 .

The composite pressure field is, using the notation given by (4),

$$
p(\mathbf{x}, t)=p_{s}\left(\mathbf{r}_{1} ; a_{1}, \mathbf{V}_{1}-\mathbf{u}_{1}\right)+p_{s}\left(\mathbf{r}_{2} ; a_{2}, \mathbf{V}_{2}-\mathbf{u}_{2}\right) .
$$

Equations (14) and (21) together completely specify the perturbation flow field, and they satisfy the same quasisteady Stokes Eq. (6).

Now the total surface force on each droplet can be calculated using (8), while observing the results given by (9) and (10), yielding

$$
\begin{aligned}
& \mathbf{F}_{1}=\rho g \frac{4 \pi a_{1}^{3}}{3} \mathbf{e}_{3}-6 \pi \mu a_{1}\left[\mathbf{V}_{1}-\mathbf{u}_{1}\right] \\
& \mathbf{F}_{2}=\rho g \frac{4 \pi a_{2}^{3}}{3} \mathbf{e}_{3}-6 \pi \mu a_{2}\left[\mathbf{V}_{2}-\mathbf{u}_{2}\right]
\end{aligned}
$$

Therefore the drag forces retain the same form except that the perturbation velocities need to be subtracted.

\section{Results and discussions}

We shall now compare different formulations of the superposition method. First, consider two cases with droplets of equal size touching each other as shown in Fig. 3. We denote the vertical touch as case 1 and horizontal touch as case 2 . At steady state, the symmetry of the Stokes flow implies that the two droplets will settle at the same velocity $V_{1}=V_{2}=V$ for both cases. For case 1 , it can be shown that the magnitudes of predicted drag force based on three different formulations are

$$
D(\text { Original formulation })=6 \pi \mu a\left[V-\frac{11}{16} V\right],
$$

$$
D(\text { Integral formulation })=6 \pi \mu a\left[V-\frac{5}{13} V\right]
$$

$D($ Center-point formulation $)=6 \pi \mu a\left[V-\frac{11}{27} V\right]$.

For case 2, the results are

$$
D(\text { Original formulation })=6 \pi \mu a\left[V-\frac{13}{32} V\right]
$$
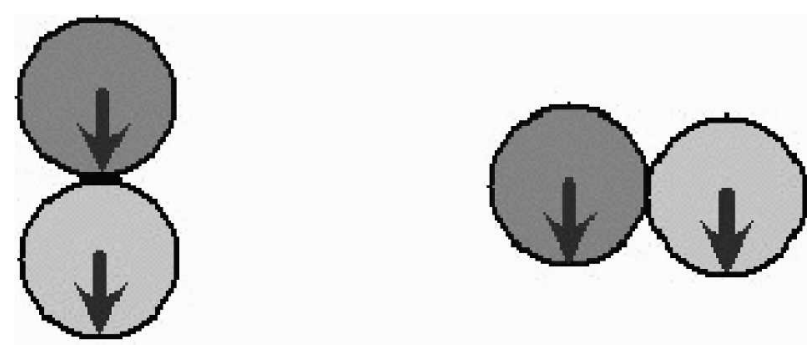

FIG. 3. Two cases with droplets touching each other: (a) case 1 -vertical alignment and (b) case 2-horizontal alignment. 


$$
\begin{aligned}
D(\text { Integral formulation }) & =6 \pi \mu a\left[V-\frac{7}{23} V\right], \\
D(\text { Center-point formulation }) & =6 \pi \mu a\left[V-\frac{13}{45} V\right] .
\end{aligned}
$$

These are compared, in Tables 1 and 2, with the exact Stokes flow solutions given by Stimson and Jeffery (1926) and Davis (1969), as well the results of Klett and Davis (1973) and Klett (1976). Several observations can be made. First the relative error in drag prediction is greatly reduced with the improved formulations, when compared to the original formulation. The very large relative errors in the original formulation of the superposition method were recognized before, and were often criticized in the literature (e.g., Klett 1976; Pruppacher and Klett 1978). We demonstrate that these large errors can be largely removed by simply imposing the boundary condition constraints in the method. Second, the integral formulation yields the exact same results as those of Klett and Davis (1973), indicating the level of approximations of our improved formulation is very much similar to that of Klett and Davis (1973). Third, the integral formulation is not necessarily more accurate than the center-point formulation; therefore, since the center-point formulation is much simpler to implement, we recommend the center-point formulation as a preferred approach.

Obviously, when the center-point formulation is used, the average fluid velocity on the surface of the droplet may not satisfy the no-slip boundary condition. For case 1, the center-point formulation yields the following average fluid velocity on the surface of the droplet:

$$
\left.\mathbf{u}(\mathbf{x}, t)\right|_{\left|\mathbf{r}_{1}\right|=a}=\frac{26}{27} \mathbf{V}=0.9630 \mathbf{V}
$$

namely, a small positive slip exists between the droplet surface and the fluid. Since the integral formulation for this case underpredicts the drag or the relative motion

TABLE 1. Comparison of predicted drag force for two droplets touching vertically.

\begin{tabular}{lcc}
\hline \multicolumn{1}{c}{ Method } & $\begin{array}{c}\text { Drag } \\
\text { force }\end{array}$ & $\begin{array}{c}\text { Relative } \\
\text { error }\end{array}$ \\
\hline $\begin{array}{l}\text { Analytical Stokes flow (Stimson and } \\
\quad \text { Jeffery 1926) }\end{array}$ & $0.645 D_{S}$ & \\
Klett and Davis (1973) in the Stokes limit & $0.615 D_{S}$ & $4.7 \%$ \\
Klett (1976) & $0.624 D_{S}$ & $3.3 \%$ \\
$\begin{array}{l}\text { Original superposition method (e.g., } \\
\quad \text { Pruppacher and Klett 1978) }\end{array}$ & $0.313 D_{S}$ & $51.5 \%$ \\
$\begin{array}{l}\text { Improved superposition method (integral } \\
\quad \text { formulation) }\end{array}$ & $0.615 D_{S}$ & $4.7 \%$ \\
$\begin{array}{l}\text { Improved superposition method (center- } \\
\quad \text { point formulation) }\end{array}$ & $0.593 D_{S}$ & $8.1 \%$ \\
\hline
\end{tabular}

TABLE 2. Comparison of predicted drag force for two droplets touching horizontally.

\begin{tabular}{lcc}
\hline \hline \multicolumn{1}{c}{ Method } & $\begin{array}{c}\text { Drag } \\
\text { force }\end{array}$ & $\begin{array}{c}\text { Relative } \\
\text { error }\end{array}$ \\
\hline $\begin{array}{l}\text { Analytical Stokes flow (Davis 1969) } \\
\text { Klett and Davis (1973) in the Stokes limit }\end{array}$ & $\begin{array}{c}0.725 D_{S} \\
0.696 D_{S}\end{array}$ & $4.0 \%$ \\
$\begin{array}{l}\text { Original superposition method (e.g., } \\
\quad \text { Pruppacher and Klett 1978) }\end{array}$ & $0.594 D_{S}$ & $18.1 \%$ \\
$\begin{array}{l}\text { Improved superposition method (integral } \\
\text { formulation) }\end{array}$ & $0.696 D_{S}$ & $4.0 \%$ \\
$\begin{array}{l}\text { Improved superposition method } \\
\text { (center-point formulation) }\end{array}$ & $0.711 D_{S}$ & $1.9 \%$ \\
\hline
\end{tabular}

used to compute the drag, this positive slip worsens the drag force representation in the center-point formulation. On the other hand, for case 2, the implied average fluid velocity on the surface of the droplet for the center-point formulation is

$$
\left.\mathbf{u}(\mathbf{x}, t)\right|_{\left|\mathbf{r}_{1}\right|=a}=\frac{46}{45} \mathbf{V}=1.0222 \mathbf{V},
$$

giving a $2 \%$ negative slip. As in case 1 , the integral formulation underpredicts the drag; this negative slip now improves the drag force representation in the center-point formulation.

To illustrate the advantages of our improved formulations, we compare the vector velocity fields observed when moving with the droplets in Fig. 4 for case 1. In the original formulation, the disturbance flow is too weak and the relative velocity field is crossing through the droplet surfaces, leading to the severe underprediction of the drag force. On the other hand, in the new formulations, the surface boundary conditions are approximately satisfied (in an average sense), leading to much more realistic flow fields. Since the drag force is also an average flow property, we obtain a rather accurate drag even though the nonslip boundary conditions are not exactly satisfied at local points on the surfaces. It should be remembered that the drag force is what matters as far as the motion of the droplets is concerned, so we would expect that our improved superposition method will yield reasonably accurate droplet trajectories for these cases discussed here. The improved formulations also show the presence of vortex flows in between the two droplets, a feature previously experimentally observed (Kumagai and Muraoka 1989).

Next we extend the above comparisons to untouching droplets by varying the separation distance between droplets. First, the case of vertical alignment is considered. In Fig. 5a, we compare the normalized drag force, plotted as a function of normalized separation distance, again assuming the droplets are of equal size and settle at the same velocity. The format of this figure is the same as Fig. 14-2 of Pruppacher and Klett (1997). The drag forces predicted using various formulations are given here as 


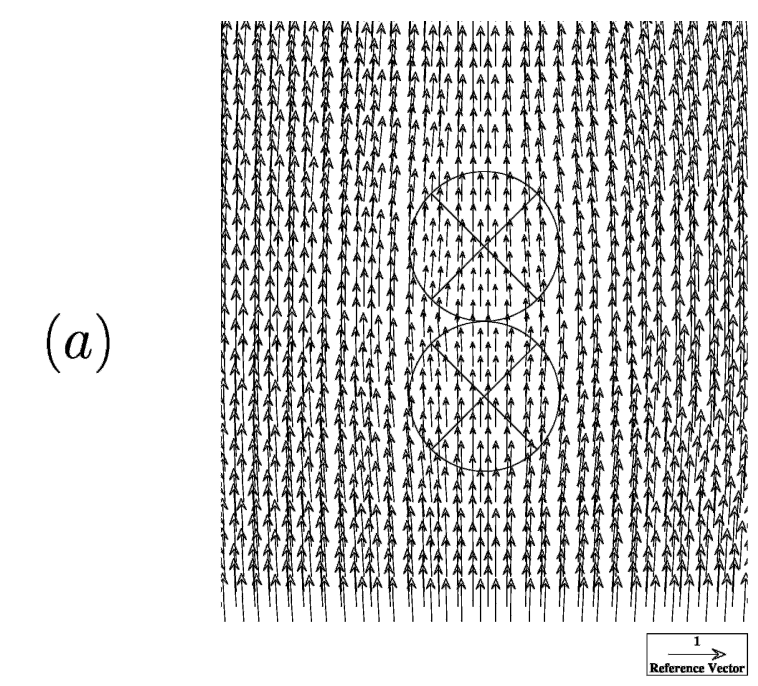

(b)

(c)

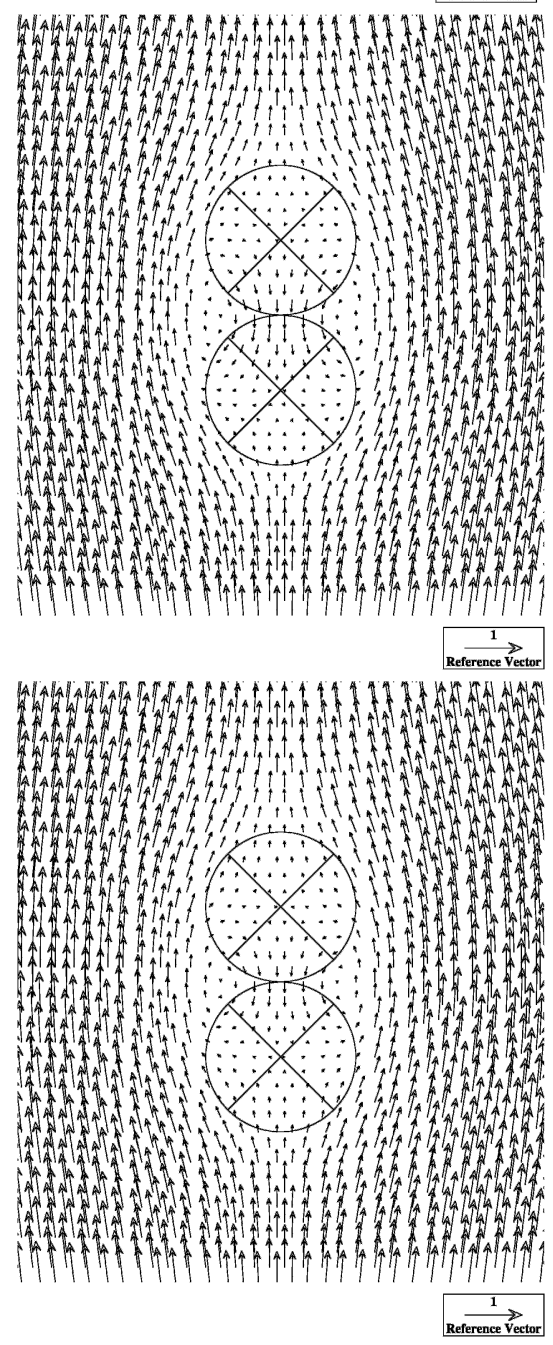

FIG. 4. Airflow realized by moving with the droplets: (a) original formulation; (b) integral formulation; and (c) center-point formulation. $(a)$

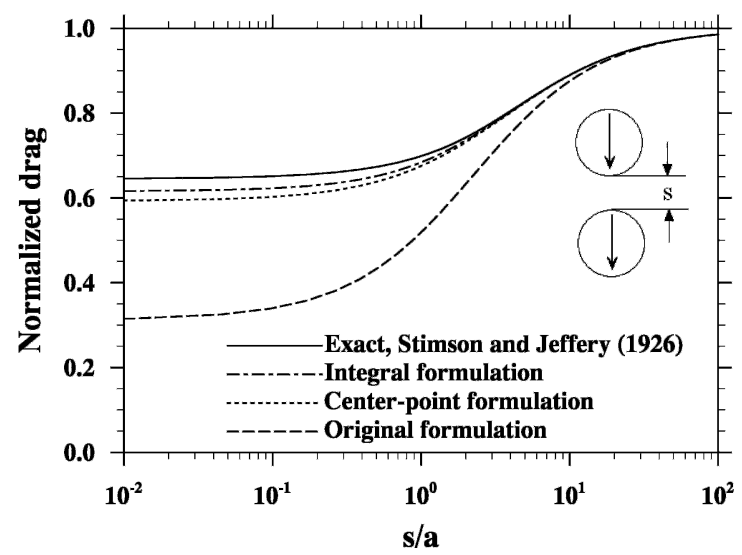

(b)

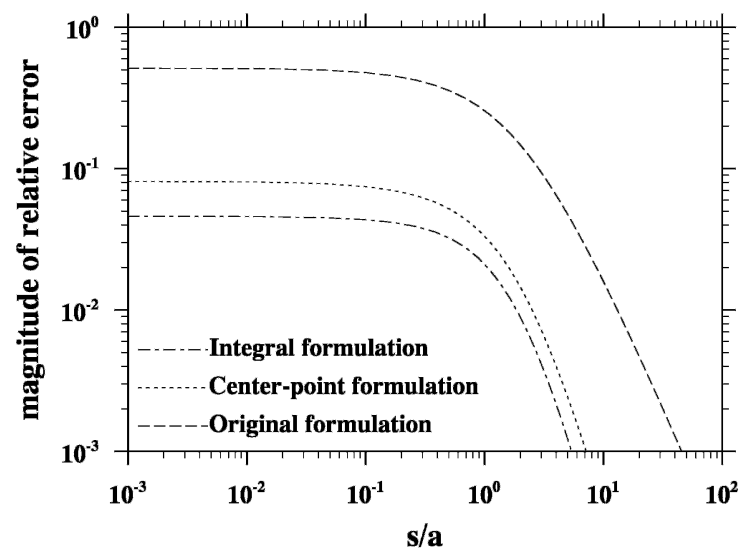

FIG. 5. Comparison of various formulations for equal parallel motion parallel to the line of centers: (a) predicted drag and (b) magnitude of relative error of drag prediction.

$$
D(\text { Original formulation })=D_{S}\left[1-1.5 \alpha+0.5 \alpha^{3}\right] \text {, }
$$

$D($ Integral formulation $)=$

$$
D_{S}\left[\frac{2\left(1-\alpha^{2}\right)}{2+3 \alpha-2 \alpha^{2}-5 \alpha^{3}+2 \alpha^{5}}\right],
$$

$D($ Center-point formulation $)=D_{S}\left[\frac{2}{2+3 \alpha-\alpha^{3}}\right]$,

where $\alpha \equiv a /(2 a+s)$, and $s$ is the separation distance as shown in the figure. The exact solution of Stimson and Jeffery (1926) is also shown:

$$
\frac{D}{D_{S}}=\frac{4}{3} \sinh \beta \sum_{n=1}^{\infty} \frac{n(n+1)}{(2 n-1)(2 n+3)}
$$

$$
\times\left\{1-\frac{4 \sinh ^{2}\left(n+\frac{1}{2}\right) \beta-(2 n+1)^{2} \sinh ^{2} \beta}{2 \sinh (2 n+1) \beta+(2 n+1) \sinh 2 \beta}\right\},
$$


where $\alpha$ and $\beta$ are related by $2 \alpha \cosh \beta=1$. Once again, our improved formulations compare well with the exact result of Stimson and Jeffery (1926). For all formulations, the deviations from the exact solution increase with decreasing separation. All formulations underpredict the drag force. The integral formulation gives better prediction than the center-point formulation for this case. The relative errors are plotted in Fig. $5 b$. The relative error drops very quickly with $s / a$ if $s / a$ exceeds one. All formulations give a relative error of less than $1 \%$ if $s / a>20$, indicating the effects of local disturbance flows are contained within $s=20 a$. Our improved formulations have a relative error of about one order of magnitude smaller than the relative error in the original formulation.

For the case of horizontal alignment and finite separation, we can obtain

$$
\begin{gathered}
D(\text { Original formulation })= \\
D_{S}\left[1-0.75 \alpha-0.25 \alpha^{3}\right] \\
D(\text { Integral formulation })= \\
D_{S}\left[\frac{4\left(1-\alpha^{2}\right)}{4+3 \alpha-4 \alpha^{2}-\alpha^{3}-2 \alpha^{5}}\right] \\
D(\text { Center-point formulation })=D_{S}\left[\frac{4}{4+3 \alpha+\alpha^{3}}\right] .
\end{gathered}
$$

These results are compared in Fig. 6a. Also plotted by symbols is the numerical result of Davis (1969) based on exact Stokes solution. For the horizontal alignment, the center-point formulation is better than the integral formulation. The relative errors are displayed in Fig. 6b. Again, our improved formulations yield a relative error that is one order of magnitude smaller than the original formulation.

The two special cases discussed above have welldefined, finite drag forces when the separation distance $s$ approaches zero. It is well known that, for Stokes flow with no-slip boundary condition, the component of the force between the droplets acting along the line of centers could increase indefinitely as $s$ approaches zero for configurations when the gap is closing due to the relative motion (Davis 1966; Hocking and Jonas 1970; Jonas 1972). Such divergent drag force is also known as the lubrication effect. The case of the antiparallel motion parallel to the line of centers shown in Fig. 7 is such an example, with the exact force acting on the droplet given as (Maude 1961)

$$
\begin{aligned}
\frac{D}{D_{S}}= & -\frac{4}{3} \sinh \beta \sum_{n=1}^{\infty} \frac{n(n+1)}{(2 n-1)(2 n+3)} \\
& \times\left\{1-\frac{4 \cosh ^{2}\left(n+\frac{1}{2}\right) \beta+(2 n+1)^{2} \sinh ^{2} \beta}{2 \sinh (2 n+1) \beta-(2 n+1) \sinh 2 \beta}\right\} .
\end{aligned}
$$

(a)

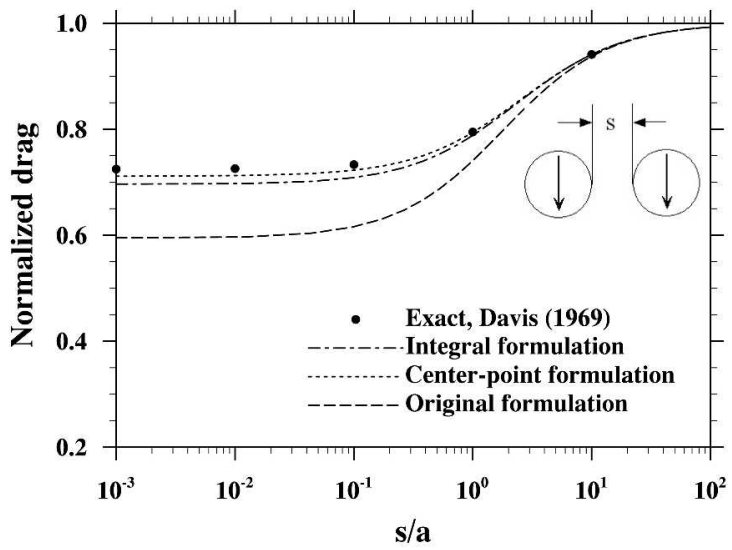

(b)

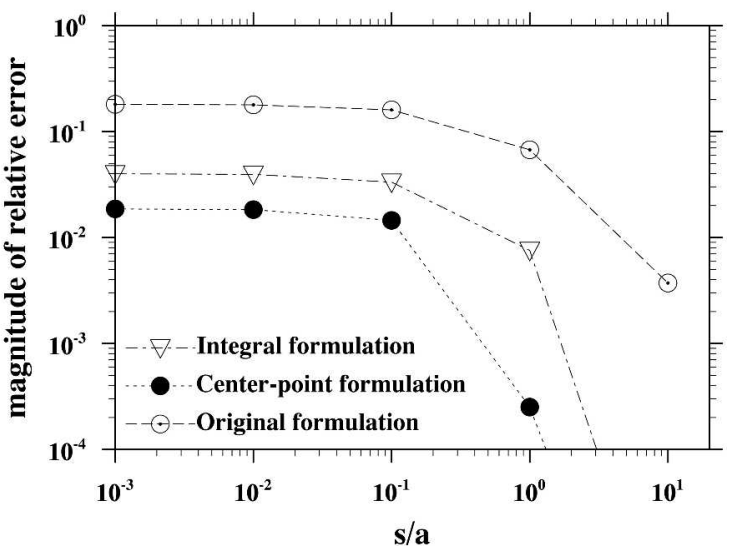

FIG. 6. Comparison of various formulations for equal parallel motion perpendicular to the line of centers: (a) predicted drag and (b) magnitude of relative error of drag prediction.

When $s \rightarrow 0$, the leading-order expansion of the above expression yields

$$
\frac{D}{D_{S}} \approx 0.5 \frac{a}{s}, \quad \text { as } \frac{s}{a} \rightarrow 0,
$$

which diverges as the gap distance goes to zero because of the lubrication effect. In Fig. 7a we compare the normalized drag force, plotted as a function of normalized separation distance, again assuming the droplets are of equal size and move at the same speed. The solid line is the same as the Stimson-Jeffery-Maude curve shown in Fig. 2 of Davis (1966). The drag forces predicted using various formulations are as follows:

$D($ Original formulation $)=$

$$
D_{S}\left[1+1.5 \alpha-0.5 \alpha^{3}\right]
$$

$D($ Integral formulation $)=$

$$
D_{S}\left[\frac{2\left(1-\alpha^{2}\right)}{2-3 \alpha-2 \alpha^{2}+5 \alpha^{3}-2 \alpha^{5}}\right] \text {, }
$$


$(a)$

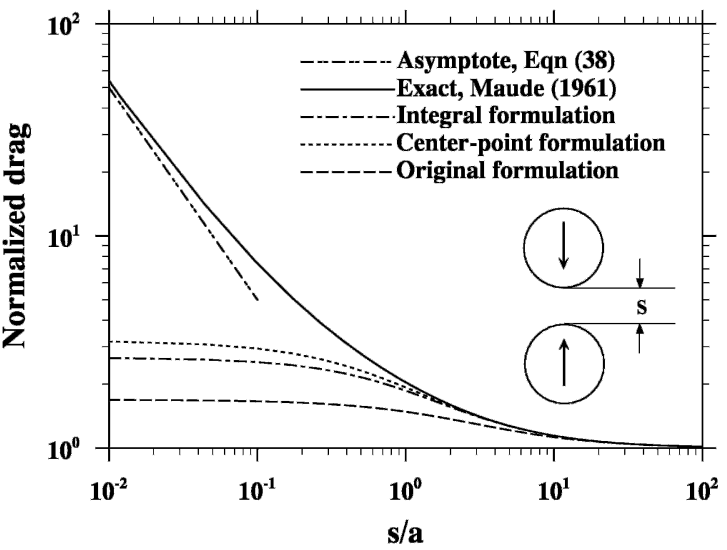

(b)

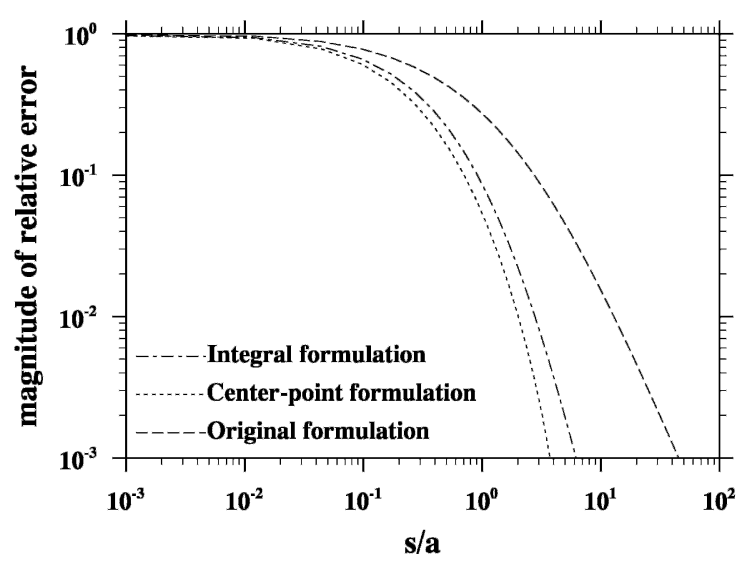

FIG. 7. Comparison of various formulations for antiparallel motion parallel to the line of centers: (a) predicted drag and (b) magnitude of relative error of drag prediction.

$$
D(\text { Center-point formulation })=D_{S}\left[\frac{2}{2-3 \alpha+\alpha^{3}}\right] .
$$

While our improved formulations still perform better than the original formulation, all the formulations based on the superposition method fail to predict the lubrication effect. The relative errors for all formulations are displayed in Fig. $7 \mathrm{~b}$, showing that even the improved formulations incur a large error when $s / a<1$. When two droplets move toward each other at small separation, the stress (local pressure and viscous shear stress) distributions on the surface of droplets may become very nonuniform, with possibly explosive growth of local stress within the region in between the two droplets, leading to extremely large normal stress acting on the droplets. Our approximate formulations will not handle such a situation accurately, as shown in Fig. 7.

Finally, we compare the collision efficiencies of cloud droplets predicted by the original superposition method and the improved method based on the center-point formulation in Fig. 8. The gravitational hydrodynamic interactions in calm air without slip-flow corrections are considered here. It was assumed that initially two droplets were separated by a center-to-center distance of $50\left(a_{1}+a_{2}\right)$ in the vertical direction. The collision efficiencies were computed by finding the far-field, offcenter horizontal separation for the grazing trajectories with an efficient midpoint algorithm. When estimates of upper and lower bounds, $E_{1}$ and $E_{2}$, were assumed, the algorithm approaches the correct off-center horizontal separation for the grazing trajectories by a factor of $1 / 2$ in each iteration. Typically, the code was run for seven iterations, so the accuracy was $\left(E_{2}-E_{1}\right) / 2^{7}=$ $0.0078\left(E_{2}-E_{1}\right)$, leading to convergence of the first two digits in collision efficiency. A second run was then done with better estimates of the bounds to converge the value of collision efficiency to four significant digits, provided that the roundoff errors are not important. Since only single precision was used and the roundoff error may become important for small $a_{1}$ and $a_{2} / a_{1}$, the data for $a_{1}=5 \mu \mathrm{m}$ and small $a_{2} / a_{1}$ may not have the same accuracy. The data based on the center-point formulation and the original formulation are also listed in Tables 3 and 4, respectively.

Since the level and influence of disturbance flows were underestimated in the original formulation of the superposition method, the collision efficiencies predicted by the original formulation are larger than those predicted by the improved formulation. The differences between the two formulations increase with decreasing droplet size and increasing size ratio. These are expected since the hydrodynamic interaction times are too small to influence the motion of droplets when droplets are large enough. Further, if the size ratio is small, the disturbance flow due to the larger droplet dominates over that due to the smaller droplet, and in that case, only the motion of the smaller droplet is affected by the hydrodynamic interaction; as such the improved formulation reduces essentially to the original formulation.

We also note that the collision efficiencies predicted by the improved formulation compare much better with the results of Davis and Sartor (1967) based on the Stokes flow solution of two-droplet hydrodynamics. In Davis and Sartor (1967), the grazing trajectories were assumed to be at a finite minimum separation of 0.001 times the radius of the larger droplet. We recalculated the collision efficiencies with such a small gap, and the results are displayed in Table 5. We find that allowing a small gap increases the collision efficiencies by less than $5 \%$ for $a_{2} / a_{1}>0.10$. When $a_{2} / a_{1} \leq 0.10$, the collision efficiencies are small, and the increases could be as much as $10 \%$. This dependence of collision efficiencies on the gap size is much weaker than what was reported by Hocking and Jonas (1970). This weaker dependence is explained by the lack of the lubrication effect in the superposition formulations as shown in Fig. 7. 


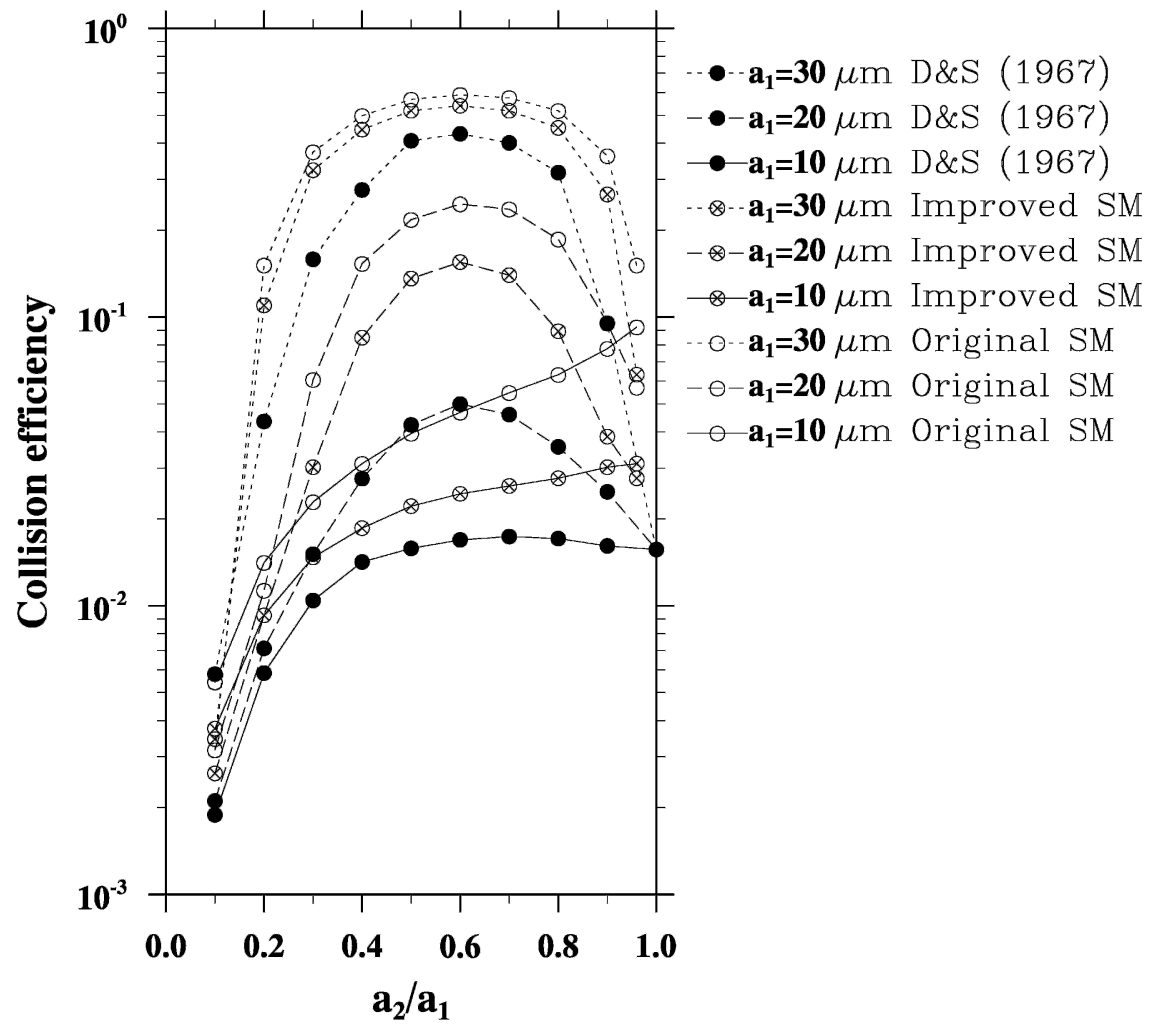

FIG. 8. Collision efficiencies predicted by the original superposition method and the improved method using the center-point formulation, compared with the results of Davis and Sartor (1967) based on Stokes flow solution of two-droplet hydrodynamics.

Our improved formulation still overpredicts the collision efficiency due to the approximations present in our simple approach, in particular, the lack of the lubrication effect.
Finally, we note that the collision efficiencies predicted by our improved superposition method compare well with the results of Klett and Davis (1973) when taking the zero-Reynolds-number limit in their formu-

TABLE 3. Calculated collision efficiencies for hydrodynamic interactions under gravity with the center-point formulation. Zero gap was used for collision detection.

\begin{tabular}{|c|c|c|c|c|c|c|}
\hline \multicolumn{7}{|c|}{ Drop radius $a_{1}(\mu \mathrm{m})$} \\
\hline$a_{2} / a_{1}$ & 5 & 10 & 15 & 20 & 25 & 30 \\
\hline$\overline{0.05}$ & 0.000000 & 0.001904 & $3.856 \times 10^{-5}$ & $2.089 \times 10^{-5}$ & $4.531 \times 10^{-5}$ & 0.001614 \\
\hline 0.10 & 0.005815 & 0.003441 & 0.003203 & 0.002216 & 0.002647 & 0.003011 \\
\hline 0.15 & 0.0002367 & 0.006000 & 0.005355 & 0.004952 & 0.006188 & 0.01515 \\
\hline 0.20 & 0.008325 & 0.008647 & 0.007646 & 0.008662 & 0.01997 & 0.1075 \\
\hline 0.25 & 0.01251 & 0.01132 & 0.01034 & 0.01560 & 0.07326 & 0.2219 \\
\hline 0.30 & 0.01553 & 0.01360 & 0.01350 & 0.02927 & 0.1464 & 0.3171 \\
\hline 0.35 & 0.01816 & 0.01572 & 0.01710 & 0.05348 & 0.2131 & 0.3903 \\
\hline 0.40 & 0.02063 & 0.01762 & 0.02105 & 0.08321 & 0.2672 & 0.4450 \\
\hline 0.45 & 0.02253 & 0.01935 & 0.02502 & 0.1109 & 0.3084 & 0.4844 \\
\hline 0.50 & 0.02431 & 0.02083 & 0.02863 & 0.1326 & 0.3374 & 0.5114 \\
\hline 0.55 & 0.02578 & 0.02213 & 0.03150 & 0.1465 & 0.3550 & 0.5275 \\
\hline 0.60 & 0.02707 & 0.02326 & 0.03324 & 0.1521 & 0.3618 & 0.5339 \\
\hline 0.65 & 0.02816 & 0.02424 & 0.03373 & 0.1488 & 0.3579 & 0.5306 \\
\hline 0.70 & 0.02903 & 0.02505 & 0.03301 & 0.1364 & 0.3421 & 0.5168 \\
\hline 0.75 & 0.02973 & 0.02581 & 0.03149 & 0.1153 & 0.3129 & 0.4903 \\
\hline 0.80 & 0.03036 & 0.02657 & 0.02953 & 0.08703 & 0.2670 & 0.4466 \\
\hline 0.85 & 0.03075 & 0.02748 & 0.02758 & 0.05763 & 0.1997 & 0.3771 \\
\hline 0.90 & 0.03146 & 0.02856 & 0.02646 & 0.03690 & 0.1101 & 0.2650 \\
\hline 0.95 & 0.03189 & 0.03004 & 0.02718 & 0.02720 & 0.03832 & 0.09249 \\
\hline
\end{tabular}


TABLE 4. Calculated collision efficiencies for hydrodynamic interactions under gravity with the original formulation. Zero gap was used for collision detection.

\begin{tabular}{|c|c|c|c|c|c|c|}
\hline$a_{2} / a_{1}$ & 5 & 10 & 15 & 20 & 25 & 30 \\
\hline 0.05 & 0.0000 & 0.003805 & 0.001427 & 0.001342 & 0.001017 & $9.039 \times 10^{-4}$ \\
\hline 0.10 & 0.006836 & 0.004830 & 0.003527 & 0.002830 & 0.002535 & 0.002845 \\
\hline 0.15 & 0.01236 & 0.008773 & 0.006128 & 0.005235 & 0.007109 & 0.02760 \\
\hline 0.20 & 0.01899 & 0.01335 & 0.008984 & 0.01036 & 0.03727 & 0.1488 \\
\hline 0.25 & 0.02675 & 0.01774 & 0.01279 & 0.02435 & 0.1189 & 0.2710 \\
\hline 0.30 & 0.03503 & 0.02193 & 0.01820 & 0.05889 & 0.2043 & 0.3680 \\
\hline 0.35 & 0.04316 & 0.02607 & 0.02595 & 0.1050 & 0.2764 & 0.4412 \\
\hline 0.40 & 0.05106 & 0.03014 & 0.03641 & 0.1493 & 0.3334 & 0.4952 \\
\hline 0.45 & 0.05838 & 0.03418 & 0.04869 & 0.1865 & 0.3761 & 0.5340 \\
\hline 0.50 & 0.06510 & 0.03812 & 0.06082 & 0.2146 & 0.4063 & 0.5607 \\
\hline 0.55 & 0.07102 & 0.04197 & 0.07075 & 0.2335 & 0.4255 & 0.5773 \\
\hline 0.60 & 0.07649 & 0.04563 & 0.07750 & 0.2430 & 0.4343 & 0.5848 \\
\hline 0.65 & 0.08125 & 0.04911 & 0.07987 & 0.2431 & 0.4332 & 0.5835 \\
\hline 0.70 & 0.08547 & 0.05260 & 0.07839 & 0.2335 & 0.4215 & 0.5729 \\
\hline 0.75 & 0.08916 & 0.05639 & 0.07350 & 0.2137 & 0.3977 & 0.5510 \\
\hline 0.80 & 0.09238 & 0.06104 & 0.06633 & 0.1830 & 0.3587 & 0.5144 \\
\hline 0.85 & 0.09518 & 0.06724 & 0.05907 & 0.1415 & 0.2994 & 0.4554 \\
\hline 0.90 & 0.09771 & 0.07611 & 0.05524 & 0.09344 & 0.2120 & 0.3587 \\
\hline 0.95 & 0.09963 & 0.08796 & 0.06323 & 0.05762 & 0.09800 & 0.1922 \\
\hline
\end{tabular}

lation, as shown in Fig. 2 of Klett and Davis (1973). Interestingly, our results are better than those of Klett and Davis (1973) for the $a_{1}=10 \mu \mathrm{m}$ case, when compared to the prediction of Davis and Sartor (1967). Therefore, it is very important to note that much of the differences between the predictions of Klett and Davis (1973) based on an approximate Oseen flow and those of Davis and Sartor (1967) based on the Stokes flow hydrodynamics are due to the approximations in Klett and Davis (1973), rather than the true finite-Reynoldsnumber correction. Similar to our formulation, Klett and Davis (1973) superimposed two Oseen flow solutions and required the composite flow to approximately satisfy the boundary conditions on the surface of two droplets. However, the detailed implementation of the boundary conditions were not clearly specified. Furthermore, since the two Oseen flows due to the two droplets satisfy different governing equations, it is not clear what the exact equation is that governs the composite flow.

On the other hand, the finite Reynolds number or finite fluid inertia does play an important role in modifying the relative motion [see, e.g., Fig. 3 of Klett and Davis (1973)], and, as shown in Klett and Davis (1973), this can lead to the asymmetry in the disturbance flows or wake effect, which results in higher collision efficiency when the radius ratios are larger than 0.8 . This physical effect is not considered at all in this study.

\section{Conclusions and remarks}

In this paper we demonstrate that the original implementation of the superposition method can be greatly improved, using a consistent framework based on the relationship between the disturbance flows and the viscous drag forces acting on droplets. Essentially, we require that the composite flow satisfies, in an average sense, the boundary conditions on the surface of droplets. Two formulations-namely, the integral formulation, and the center-point formulation-are possible. To illustrate the accuracy of the improved formulations, we have considered hydrodynamic interactions

TABLE 5. Calculated collision efficiencies for hydrodynamic interactions under gravity with the center-point formulation. A small gap of $0.001 a_{1}$ was used for collision detection.

\begin{tabular}{lcccccc}
\hline \hline \multicolumn{7}{c}{ Drop radius $a_{1}(\mu \mathrm{m})$} \\
\hline$a_{2} / a_{1}$ & 5 & 10 & 15 & 20 & \multicolumn{1}{c}{25} & 0.002849 \\
\hline 0.10 & 0.005832 & 0.003646 & 0.003444 & 0.002452 & 0.002068 & 0.1083 \\
0.20 & 0.008527 & 0.009013 & 0.008000 & 0.009058 & 0.1471 & 0.3180 \\
0.30 & 0.01596 & 0.01404 & 0.01394 & 0.02998 & 0.2681 & 0.4459 \\
0.40 & 0.02113 & 0.01807 & 0.02157 & 0.08402 & 0.3382 & 0.5122 \\
0.50 & 0.02479 & 0.02127 & 0.02919 & 0.1334 & 0.3626 & 0.5347 \\
0.60 & 0.02747 & 0.02370 & 0.03379 & 0.1528 & 0.3428 & 0.5175 \\
0.70 & 0.02937 & 0.02550 & 0.03354 & 0.1371 & 0.2676 & 0.4473 \\
0.80 & 0.03077 & 0.02698 & 0.02996 & 0.08769 & 0.1107 & 0.2656 \\
0.90 & 0.03178 & 0.02893 & 0.02685 & 0.03745 & & \\
\hline
\end{tabular}


between two droplets under Stokes disturbance flow approximations. Results on viscous drag force compare favorably with known exact solutions of Stokes flows. Specifically, the relative errors on the drag force can be reduced by one order of magnitude using the improved formulations, when compared with the original formulation, for situations when the lubrication effect is not dominant.

We also show that the improved formulation results in a better prediction of collision efficiencies. However, like previous approximate methods such as Klett and Davis (1973), our improved formulation still overestimates the collision efficiencies, by a factor of as much as 2 to 3 . The level of accuracy of our improved superposition method is comparable to that of Klett and Davis (1973) when the Stokes disturbance flows are considered. The advantage of our formulation is its simplicity of implementation. Another advantage is that our method can be applied to any droplet-droplet, threedimensional relative configurations in space, while extension of Klett and Davis's (1973) formulation to general three-dimensional configurations has not yet been worked out.

We note that the concepts explored in this paper could be further developed to describe disturbance flows at finite droplet Reynolds numbers, and we will report on this aspect in the future. Previous results (Klett and Davis 1973) suggest that Stokes disturbance flows could underestimate collision efficiencies, particularly when the droplets are of similar size. Therefore, our improved formulations must be further developed to include the finite-Reynolds-number effect in order to better quantify the collision efficiencies of cloud droplets. At this stage, the results of Klett and Davis (1973) on collision efficiency could still be more accurate (in a qualitative sense) than the Stokes-flowbased formulations discussed here, especially for droplets of similar size. The finite-Reynolds-number effect on droplet-droplet relative motion has been demonstrated experimentally (e.g., Steinberger et al. 1968; Kumagai and Muraoka 1989). The work of Klett and Davis (1973) represents an important step toward including the finite-Reynolds-number effect in the calculation of collision efficiency; however, we feel that their formulation needs to be reexamined in light of the results discussed in this paper. The ultimate conclusion will rest on further knowledge based on a firstprinciple-based solution of droplet-droplet hydrodynamic interactions, such as direct simulations or carefully designed experimental measurements of two or more droplets in viscous fluid at finite Reynolds numbers, as exact analytical solutions have not been proven possible at finite Reynolds numbers.

Our improved formulations can be easily extended to study hydrodynamic interactions among a large number of droplets in a turbulent flow. This was our motivation to revisit the superposition technique in the first place.
Results from numerical simulations applying the improved superposition method for a large number of droplets in gravitational settling only, as well as in the turbulent particle-laden flows, are being reported in separate papers (Wang et al. 2004, manuscript submitted to J. Fluid Mech.; Wang et al. 2005).

Finally, we would like to briefly comment on the implications of the different collision efficiencies on the time scale of spectral broadening for droplet size distribution. The local time scale for spectral broadening is inversely proportional to collision efficiency. For an initially narrow size distribution of droplets around 10 to $20 \mu \mathrm{m}$, we have shown here that the calculated collision efficiencies based on different formulations can differ by a factor of 2 to 5 , indicating a significant uncertainty in predicting the time scale for spectral broadening. When the background air turbulence is considered, preliminary results Wang et al. (2005) show that air turbulence can increase the collision efficiency by as much as $50 \%$, leading to shortening of the time needed to produce droplets of $50 \mu \mathrm{m}$ or larger in radius for which the gravitational coalescence can then become very effective.

Acknowledgments. This study has been supported by the National Science Foundation through Grant ATM0114100 and by the National Center for Atmospheric Research (NCAR). LPW thanks Dr. Charmaine Franklin of McGill University for providing us the reference to the vector form of the Stokes flow solution. OA is grateful to the additional computing resources provided by the Scientific Computing Division at NCAR.

\section{REFERENCES}

Beard, K. V., and S. N. Grover, 1974: Numerical collision efficiencies for small raindrops colliding with micron size particles. J. Atmos. Sci., 31, 543-550.

Davis, M. H., 1966: Collisions of very small cloud drops. J. Geophys. Res., 71, 3101-3104.

- 1969: The slow translation and rotation of two unequal spheres in a viscous fluid. Chem. Eng. Sci., 24, 1769-1776.

— , and J. D. Sartor, 1967: Theoretical collision efficiencies for small cloud droplets in Stokes flow. Nature, 215, 1371-1372.

Hocking, L. M., 1973: Effect of slip on motion of a sphere close to a wall and of 2 adjacent spheres. J. Eng. Math., 7, 207-221.

— , and P. R. Jonas, 1970: The collision efficiency of small drops. Quart. J. Roy. Meteor. Soc., 96, 722-729.

Jonas, P. R., 1972: The collision efficiency of small drops. Quart. J. Roy. Meteor. Soc., 98, 681-683.

Kim, S., and S. J. Karrila, 1991: Microhydrodynamics: Principles and Selected Applications. Butterworth-Heinemann, 507 pp.

Klett, J. D., 1976: Comments on "Collision efficiency of water drops in the atmosphere." J. Atmos. Sci., 33, 870-872.

_ and M. H. Davis, 1973: Theoretical collision efficiencies of cloud droplets at small Reynolds numbers. J. Atmos. Sci., 30, 107-117.

Kumagai, T., and M. Muraoka, 1989: On the motion of spheres in a fluid at low Reynolds numbers (two equal-sized spheres moving along their center lines). JSME Int. J. I Series II, 32, 309-316.

Langmuir, I., 1948: The production of rain by a chain reaction in 
cumulus clouds at temperatures above freezing. J. Meteor., 5, 175-191.

Lin, C., and S. Lee, 1975: Collision efficiency of water drops in the atmosphere. J. Atmos. Sci., 32, 1412-1418.

Maude, A. D., 1961: End effects in a falling-sphere viscometer. $\mathrm{Br}$. J. Appl. Phys., 12, 293-295.

Neiburger, M., 1967: Collision efficiency of nearly equal cloud drops. Mon. Wea. Rev., 95, 917-920.

Pinsky, M., A. Khain, and M. Shapiro, 1999: Collisions of small drops in a turbulent flow. Part I: Collision efficiency. Problem formulation and preliminary results. J. Atmos. Sci., 56, 25852600.

Pruppacher, H. R., and J. D. Klett, 1978: Microphysics of Clouds and Precipitation. D. Reidel, 714 pp.
- and —, 1997: Microphysics of Clouds and Precipitation. 2d ed. Kluwer Academic, 954 pp.

Shafrir, U., and M. Neiburger, 1963: Collision efficiencies of two spheres falling in a viscous medium. J. Geophys. Res., 68, 4141-4147.

Steinberger, E. H., H. R. Pruppacher, and M. Neiburger, 1968: On the hydrodynamics of spheres falling along their line of centers in a viscous medium. J. Fluid Mech., 34, 809-819.

Stimson, M., and G. B. Jeffery, 1926: The motion of two spheres in a viscous fluid. Proc. Roy. Soc. London, A111, 110-117.

Wang, L.-P., O. Ayala, S. E. Kasprzak, and W. W. Grabowski, 2005: Theoretical formulation of collision rate and collision efficiency of hydrodynamically interacting cloud droplets in turbulent atmospheres. J. Atmos. Sci., in press. 